ISSN 1561-2430 (Print)

ISSN 2524-2415 (Online)

UDC 519.237

https://doi.org/10.29235/1561-2430-2021-57-2-206-216

Received 15.04.2021

Поступила в редакцию 15.04.2021

\author{
Vladimir S. Mukha, Nancy Forat Kako \\ Belarusian State University of Informatics and Radioelectronics, Minsk, Belarus
}

\title{
THE INTEGRALS AND INTEGRAL TRANSFORMATIONS CONNECTED WITH THE JOINT VECTOR GAUSSIAN DISTRIBUTION
}

\begin{abstract}
In many applications it is desirable to consider not one random vector but a number of random vectors with the joint distribution. This paper is devoted to the integral and integral transformations connected with the joint vector Gaussian probability density function. Such integral and transformations arise in the statistical decision theory, particularly, in the dual control theory based on the statistical decision theory. One of the results represented in the paper is the integral of the joint Gaussian probability density function. The other results are the total probability formula and Bayes formula formulated in terms of the joint vector Gaussian probability density function. As an example the Bayesian estimations of the coefficients of the multiple regression function are obtained. The proposed integrals can be used as table integrals in various fields of research.

Keywords: Bayesian estimations, joint vector Gaussian distribution, multivariate integrals, total probability formula, Bayes formula, multiple regression function.

For citation. Mukha V. S., Kako N. F. The integrals and integral transformations connected with the joint vector Gaussian distribution. Vestsi Natsyianal'nai akademii navuk Belarusi. Seryia fizika-matematychnykh navuk = Proceedings of the National Academy of Sciences of Belarus. Physics and Mathematics series, 2021, vol. 57, no. 2, pp. 206-216. https://doi. org/10.29235/1561-2430-2021-57-2-206-216
\end{abstract}

\section{В. С. Муха, Н. Ф. Како}

Белорусский государственный университет информатики и радиоэлектроники, Минск, Беларусь

\section{ИНТЕГРАЛЫ И ИНТЕГРАЛЬНЫЕ ПРЕОБРАЗОВАНИЯ, СВЯЗАННЫЕ С СОВМЕСТНЫМ ВЕКТОРНЫМ ГАУССОВСКИМ РАСПРЕДЕЛЕНИЕМ}

\begin{abstract}
Аннотация. Во многих приложениях желательно рассматривать не один случайный вектор, а набор случайных векторов с совместным распределением. Данная статья посвящена интегралам и интегральным преобразованиям, связанным с совместной векторной гауссовской функцией плотности вероятности. Такие интегралы и преобразования возникают в теории статистических решений, в частности в теории дуального управления, которая базируется на теории статистических решений. Одним из представленных результатов является интеграл от совместной векторной гауссовской функции плотности вероятности. Другие результаты - это формула полной вероятности и формула Байеса, сформулированные в терминах совместной векторной гауссовской функцией плотности вероятности. В качестве примера получены байесовские оценки коэффициентов множественной функции регрессии. Предложенные интегралы могут быть использованы как табличные интегралы в различных областях исследований

Ключевые слова: байесовские оценки, совместное векторное гауссовское распределение, многомерные интегралы, формула полной вероятности, формула Байеса, множественная функция регрессии.

Для цитирования. Муха, В. С. Интегралы и интегральные преобразования, связанные с совместным векторным гауссовским распределением / В. С. Муха, Н. Ф. Како // Вес. Нац. акад. навук Беларусі. Сер. фіз.-мат. навук. 2021. - T. 57, № 2. - C. 206-216. https://doi.org/10.29235/1561-2430-2021-57-2-206-216
\end{abstract}

Introduction. The integrals connected with probability distributions are used in many applications, one of them being the statistical decision theory, or, in other words, the Bayesian approach in statistics [1-4]. The statistical decision theory attracts much attention due to the ability to formulate problems in strict mathematical form and to process data in real time. One of the technical problems solved by the statistical decision theory is the dual control problem [5]. The equations of dynamic programming in the dual control problem contain the integrals connected with the multivariate probability distributions. The integrals and integral transformations connected with the vector Gaussian distribution were considered in papers [6,7]. However, it is desirable to consider not one separate random vector, but a number of ran-

(C) Mukha V. S., Kako N. F., 2021 
dom vectors. In this paper, the results of works $[6,7]$ are generalized for the case of the joint Gaussian distribution.

1. Integrals connected with the joint vector Gaussian distribution. The random $k_{\Xi}$-component vector $\Xi^{T}=\left(\Xi_{1}, \ldots, \Xi_{k \Xi}\right)$ is distributed by the Gaussian (or normal) law if its probability density function has the following form:

$$
f(\xi)=\frac{1}{\sqrt{(2 \pi)^{k \Xi}\left|d_{\Xi}\right|}} \exp \left(-\frac{1}{2}\left(\xi-v_{\Xi}\right)^{T} d \Xi\left(\xi-v_{\Xi}\right)\right),
$$

where $\xi^{T}=\left(\xi_{1}, \ldots, \xi_{k_{\Xi}}\right)$ is the row vector of the arguments, $v_{\Xi}^{T}=\left(v_{\Xi_{1}}, \ldots, v_{\Xi_{k \Xi}}\right)$ is the row vector of the parameters, $d_{\Xi}=\left(d_{\Xi, i, j}\right), i, j=\overline{1, k_{\Xi}}$, is the symmetric positive definite $\left(k_{\Xi} \times k_{\Xi}\right)$-matrix of the parameters, $d_{\Xi}^{-1}$ is the matrix inverse to the $d_{\Xi},\left|d_{\Xi}\right|$ is the determinant of the matrix $d_{\Xi}$, and $T$ is the transpose symbol. The parameters $v_{\Xi}$ and $d_{\Xi}$ are the mathematical expectation and variance-covariance matrix of the random vector $\Xi$ respectively.

The following integral connected with function (1) was proved in [6]:

$$
\int_{E^{k \Xi}} \exp \left(-\frac{1}{2} \xi^{T} A \xi+B^{T} \xi\right) d \xi=\sqrt{(2 \pi)^{k \Xi} \mid A^{-1}} \mid \exp \left(\frac{1}{2} B^{T} A^{-1} B\right),
$$

where $\xi^{T}=\left(\xi_{1}, \ldots, \xi_{k_{\Xi}}\right), A=\left(a_{i, j}\right), i, j=\overline{1, k_{\Xi}}$, is the symmetric positive definite matrix, $B^{T}=\left(B_{1}, \ldots, B_{k \Xi}\right)$ is the row vector, $A^{-1}$ is the matrix inverse to the $A,\left|A^{-1}\right|$ is the determinant of the matrix $A^{-1}$, and $E^{k \Xi}$ is the $k_{\Xi}$-dimensional Euclidean space.

In some cases, it is desirable to consider not one random vector with the Gaussian distribution, but several random vectors with the joint Gaussian distribution. This case is possible to study by partitioning the vector $\xi^{T}=\left(\xi_{1}, \xi_{2}, \ldots, \xi_{k \Xi}\right)$ with $k_{\Xi}$ components into $m$ vectors with $k_{1}, k_{2}, \ldots, k_{m}$ components, $k_{1}+k_{2}+\ldots+k_{m}=k_{\Xi}$, so that the vector $\xi^{T}$ in (1) is $\xi^{T}=\left(\xi_{1}^{T}, \xi_{2}^{T}, \ldots, \xi_{m}^{T}\right)$, where $\xi_{1}^{T}, \xi_{2}^{T}, \ldots, \xi_{m}^{T}$ are the row vectors. Let as partition also the vector $v_{\Xi}^{T}=\left(v_{\Xi_{1}}, \ldots, v_{\Xi_{k \Xi}}\right)$ in (1) into $m$ vectors with $k_{1}, k_{2}, \ldots, k_{m}$ components, so that $v_{\Xi}^{T}=\left(v_{\Xi, 1}^{T}, v_{\Xi, 2}^{T}, \ldots, v_{\Xi, k \Xi}^{T}\right)$, and partition the rows and columns of the matrix $d \Xi$ into $m$ groups of rows and columns, agreed with the partitioning of the vectors $\xi^{T}$ and $v_{\Xi}^{T}$, so that $d_{\Xi}^{-1}=\left(d_{\Xi}^{i, j}\right), i, j=\overline{1, m}$, where $d_{\Xi}^{i, j}$ are blocks of the matrix $d \Xi$. As a result, we get the following expression instead of formula (1):

$$
\begin{gathered}
f\left(\xi_{1}, \xi_{2}, \ldots, \xi_{m}\right)=\frac{1}{\sqrt{(2 \pi)^{k \Xi}\left|d_{\Xi}\right|}} \exp \left(-\frac{1}{2} \sum_{i=1}^{m} \sum_{j=1}^{m}\left(\xi_{i}-v_{\Xi, i}\right)^{T} d_{\Xi}^{i, j}\left(\xi_{j}-v_{\Xi, j}\right)\right)= \\
=\frac{1}{\sqrt{(2 \pi)^{k \Xi}\left|d_{\Xi}\right|}} \exp \left(-\frac{1}{2} \sum_{i=1}^{m} \sum_{j=1}^{m} \xi_{i}^{T} d_{\Xi}^{i, j} \xi_{j}+\sum_{j=1}^{m} \sum_{i=1}^{m} v_{\Xi, i}^{T} d_{\Xi}^{i, j} \xi_{j}-\frac{1}{2} \sum_{i=1}^{m} \sum_{j=1}^{m} v_{\Xi}^{T} d_{\Xi}^{i, j} v_{\Xi, j}\right) .
\end{gathered}
$$

We will call expression (3) as the joint Gaussian probability density function of the random vectors $\Xi_{1}, \Xi_{2}, \ldots, \Xi_{m}$. It is supposed that the matrix $d_{\Xi}=\left(d_{\Xi, i, j}\right), i, j=\overline{1, m}$, in (1) is partitioned into the blocks corresponding to the blocks of the matrix $d_{\Xi}^{-1}$, so that the $d_{\Xi, i, i}$ is the variance-covariance matrix of the random vector $\Xi_{i}$, and the $d_{\Xi, i, j}, i \neq j$, is the covariance matrix of the random vectors $\Xi_{i}, \Xi_{j}$. The $v_{\Xi, i}$ is the mathematical expectation of the random vector $\xi_{i}$.

Let us formulate the task of generalizing formula (2) to the case of many vector variables, i. e. the task of calculating the integral connected with function (3). More specificly, let it be required to calculate the following integral:

$$
\int_{E^{k \Xi}} \exp \left(-\frac{1}{2} \xi^{T} A \xi+B^{T} \xi\right) d \xi
$$

provided the matrix $A$ is the symmetric positive definite and the vector $\xi^{T}$ consists of $m$ vectors. 
In order to solve this task, we partition the $k_{\Xi^{-}}$-component vectors $\xi^{T}$ and $B^{T}$ into $m$ vectors with $k_{1}, k_{2}, \ldots, k_{m}$, components, $k_{1}+k_{2}+\ldots+k_{m}=k$, i. e. represent them in the forms of $\xi^{T}=\left(\xi_{1}^{T}, \xi_{2}^{T}, \ldots, \xi_{m}^{T}\right)$ and $B^{T}=\left(B_{1}^{T}, B_{2}^{T}, \ldots, B_{m}^{T}\right)$ so that the vectors $\xi_{i}^{T}$ and $B_{i}^{T}$ consist of the same numbers of components. We partition also the rows of the matrix $A=\left(a_{i, j}\right), i, j=\overline{1, k_{\Xi}}$, into $m$ groups of rows and the columns into $m$ groups of columns, coordinated with the partition of the vectors $\xi^{T}$ and $B^{T}$. As a result, we obtain the matrix with block elements $A_{i, j}$ (the so-called block matrix), which we will denote

$$
A=\left(A_{i, j}\right), \quad i, j=\overline{1, m} \text {. }
$$

Let the following integral be subjected to the following calculation:

$$
\int_{E^{k \Xi}} \exp \left(-\frac{1}{2} \xi^{T} A \xi+B^{T} \xi\right) d \xi=\int_{E^{k \Xi}} \exp \left(-\frac{1}{2} \sum_{i=1}^{m} \sum_{j=1}^{m} \xi_{i}^{T} A_{i, j} \xi_{j}+\sum_{i=1}^{m} B_{i}^{T} \xi_{i}\right) d \xi_{1} \cdots d \xi_{m} .
$$

Let us proceed to the calculation of integral (6).

The application to the block matrix $A=\left(A_{i, j}\right)(5)$ of the generalized (block) Gauss algorithm [8] gives us the following block upper triangular matrix:

$$
G=\left(\begin{array}{cccc}
A_{1,1}^{(0)} & A_{1,2}^{(0)} & \cdots & A_{1, m}^{(0)} \\
0 & A_{2,2}^{(1)} & \cdots & A_{2, m}^{(1)} \\
\cdots & \cdots & \cdots & \cdots \\
0 & 0 & \cdots & A_{m, m}^{(m-1)}
\end{array}\right)=\left(A_{i, j}^{(i-1)}\right), \quad i, j=\overline{1, m} .
$$

If $i>j$ then the blocks $A_{i, j}^{(i-1)}$ in (7) is qual to zero $\left(A_{i, j}^{(i-1)}=0\right)$. The determinant of the block upper triangular matrix $G(7)$ is equal to the product of the determinants of the diagonal blocks and the same as the determinant of the matrix $A[8]$ :

$$
|G|=\left|A_{1,1}^{(0)} \| A_{2,2}^{(1)}\right| \cdots\left|A_{m, m}^{(m-1)}\right|=|A|=\prod_{i=1}^{m}\left|A_{i, i}^{(i-1)}\right| .
$$

The matrix $A$ can be represented in the form of

$$
A=G^{T} \hat{D} G
$$

where $\widehat{D}$ is the block diagonal matrix

$$
\widehat{D}=\operatorname{diag}\left\{\left(A_{1,1}^{(0)}\right)^{-1},\left(A_{2,2}^{(1)}\right)^{-1}, \cdots,\left(A_{m, m}^{(m-1)}\right)^{-1}\right\} .
$$

We denote the diagonal blocks of the matrix $\widehat{D}(9)$ as $\widehat{D}_{i, i}=\left(A_{i, i}^{(i-1)}\right)^{-1}, i=\overline{1, m}$. The following equalities are fulfilled:

$$
\left|\widehat{D}^{-1}\right|=|G|=|A|=\prod_{i=1}^{m}\left|A_{i, i}^{(i-1)}\right| .
$$

Let us suppose that we can find in the block form the inverse matrix $G^{-1}=\left(G^{i, j}\right), i, j=\overline{1, m}$. The replacing of variables

$$
x=G^{-1} z
$$
transforms the integrated function $F(x)=\exp \left(-\frac{1}{2} \xi^{T} A \xi+B^{T} \xi\right)$ in (4) to the following function of the
argument $z$ :

$$
F(z)=\exp \left(-\frac{1}{2} z^{T} P z+D z\right)
$$


where

$$
\begin{gathered}
P=\left(G^{-1}\right)^{T} A G^{-1}, \\
D=B^{T} G^{-1}=\left(D_{i}\right)=\left(\sum_{j=1}^{m} B_{j}^{T} G^{j, i}\right), \quad i=\overline{1, m} .
\end{gathered}
$$

Since, given (8),

$$
P=\left(G^{-1}\right)^{T} A G^{-1}=\left(G^{-1}\right)^{T} G^{T} \hat{D} G G^{-1}=\hat{D},
$$

we have the following function of the argument $z=\left(z_{i}\right), i=\overline{1, m}\left(z_{i}\right.$ are vectors):

$$
F(z)=\exp \left(-\frac{1}{2} z^{T} \hat{D} z+D z\right)
$$

As it is known, the following equality is fulfilled when the variables are replaced:

$$
\int F(x) d x=J \int F(z) d z,
$$

where $J$ is the Jacobian of the transformation (11):

$$
J=\left|G^{-1}\right|=|\widehat{D}|=\prod_{i=1}^{m}\left|\left(A_{i, i}^{(i-1)}\right)^{-1}\right| .
$$

Let us rewrite function $F(z)(13)$ as the function of the elements of its matrices:

$$
\begin{gathered}
F(z)=\exp \left(-\frac{1}{2} z^{T} \hat{D} z+D z\right)=\exp \left(-\frac{1}{2} \sum_{i=1}^{m} z_{i}^{T}\left(A_{i, i}^{(i-1)}\right)^{-1} z_{i}+\sum_{i=1}^{m} D_{i} z_{i}\right)= \\
=\prod_{i=1}^{m} \exp \left(-\frac{1}{2} z_{i}^{T}\left(A_{i, i}^{(i-1)}\right)^{-1} z_{i}+D_{i} z_{i}\right) .
\end{gathered}
$$

Substituting (15) into (14), we obtain

$$
\int_{E^{k \Xi}} F(x) d x=J \prod_{i=1}^{m} \int_{E^{k \Xi}} \exp \left(-\frac{1}{2} z_{i}^{T}\left(A_{i, i}^{(i-1)}\right)^{-1} z_{i}+D_{i} z_{i}\right) d z_{i} .
$$

The integral in the right-hand side of expression (16) is the integral of type (2). In such a case

$$
\int_{E^{k \Xi}} \exp \left(-\frac{1}{2} z_{i}^{T}\left(A_{i, i}^{(i-1)}\right)^{-1} z_{i}+D_{i} z_{i}\right) d z_{i}=\sqrt{(2 \pi)^{k_{i}}\left|\left(A_{i, i}^{(i-1)}\right)\right|} \exp \left(\frac{1}{2} D_{i} A_{i, i}^{(i-1)} D_{i}^{T}\right) .
$$

Substituting (17) into (16), we obtain the following formula

$$
\begin{gathered}
\int_{E^{k \Xi}} \exp \left(-\frac{1}{2} \xi^{T} A \xi+B^{T} \xi\right) d \xi=\int_{E^{k \Xi}} \exp \left(-\frac{1}{2} \sum_{i=1}^{m} \sum_{j=1}^{m} \xi_{i}^{T} A_{i, j} \xi_{j}+\sum_{i=1}^{m} B_{i}^{T} \xi_{i}\right) d \xi_{1} \cdots d \xi_{m}= \\
=\prod_{i=1}^{m} \sqrt{(2 \pi)^{k_{i}} \mid\left(A_{i, i}^{(i-1)}\right)^{-1}} \mid \exp \left(\sum_{i=1}^{m} \frac{1}{2} D_{i} A_{i, i}^{(i-1)} D_{i}^{T}\right)
\end{gathered}
$$

or in block-matrix form

$$
\int_{E^{k \Xi}} \exp \left(-\frac{1}{2} \xi^{T} A \xi+B^{T} \xi\right) d \xi=\sqrt{(2 \pi)^{k \Xi} \mid \widehat{D}^{-1}} \exp \left(\frac{1}{2} D \widehat{D}^{-1} D^{T}\right) .
$$

Let us come back in (18) from the matrices $D$ and $\bar{D}$ to the matrices $A$ and $B$. Since $|D|=\left|A^{-1}\right|$ (formula (10)), $\widehat{D}^{-1}=G A^{-1} G^{T}$ (formula (8)) and $D=B^{T} G^{-1}$ (formula (12)), then 


$$
D \widehat{D}^{-1} D^{T}=B^{T} G^{-1} G A^{-1} G^{T}\left(G^{-1}\right)^{T} B=B^{T} A^{-1} B,
$$

and instead of (18) we get the following expression:

$$
\begin{gathered}
\int_{E^{k \Xi}} \exp \left(-\frac{1}{2} \xi^{T} A \xi+B^{T} \xi\right) d \xi=\int_{E^{k \Xi}} \exp \left(-\frac{1}{2} \sum_{i=1}^{m} \sum_{j=1}^{m} \xi_{i}^{T} A_{i, j} \xi_{j}+\sum_{i=1}^{m} B_{i}^{T} \xi_{i}\right) d \xi_{1} \cdots d \xi_{m}= \\
=\sqrt{(2 \pi)^{k \Xi} \mid A^{-1}} \exp \left(\frac{1}{2} B^{T} A^{-1} B\right)=\sqrt{(2 \pi)^{k \Xi} \mid A^{-1}} \mid \exp \left(\frac{1}{2} \sum_{i=1}^{m} \sum_{j=1}^{m} B_{i}^{T} A^{i, j} B_{j}\right) .
\end{gathered}
$$

It should be taken into account that the matrices $A$ and $B$ in (19) are block matrices, i. e. $A=\left(A_{i, j}\right), B=\left(B_{i}\right)$, $i, j=\overline{1, m}$, and one deals with the block operations of inversion and of multiplication.

Let us summarize the obtained result in the form of the following theorem.

The or e $\mathrm{m} 1$ (the integral connected with the joint Gaussian distribution of the random vectors). If $A=\left(A_{i, j}\right), i, j=\overline{1, m}$, is the square block symmetric positive definite matrix, $A^{-1}=\left(A^{i, j}\right)$ is the block matrix inverse to the matrix $A=\left(A_{i, j}\right),\left|A^{-1}\right|$ is the determinant of the matrix $A^{-1}, \xi^{T}=\left(\xi_{1}^{T}, \xi_{2}^{T}, \ldots, \xi_{m}^{T}\right)$ is the vector composed of vectors $\xi_{1}^{T}, \xi_{2}^{T}, \ldots, \xi_{m}^{T}$ and permitting the block multiplication $A \xi, k_{i}$ is the number of the scalar components of the vector $\xi_{i}, k_{1}+k_{2}+\ldots+k_{m}=k, B^{T}=\left(B_{1}^{T}, B_{2}^{T}, \ldots, B_{m}^{T}\right)$ is the vector of the parameters composed of vectors $B_{1}^{T}, B_{2}^{T}, \ldots, B_{m}^{T}$ and permitting the block multiplication $B_{i}^{T} \xi_{i}$, then equalities (19) are fulfilled.

\section{Total probability formulae for the joint vector Gaussian distribution.}

Theorem 2 (total probability formula for the joint vector Gaussian distribution). Let $\Xi^{T}=\left(\Xi_{1}^{T}, \Xi_{2}^{T}, \ldots, \Xi_{m}^{T}\right)$ be a random vector composed of vectors $\Xi_{1}^{T}, \Xi_{2}^{T}, \ldots, \Xi_{m}^{T}, k_{\Xi, i}$ the number of the scalar components of the vector $\Xi_{i}, i=\overline{1, m}, f(\xi)$ the probability density function of the vector $\Xi^{T}$, $k_{\Xi}=k_{\Xi, 1}+k_{\Xi, 2}+\ldots+k_{\Xi, m}$ the number of the scalar components of the vector $\Xi, f(y / \xi)$ the conditional probability density function of the random vector $Y$, $k_{Y}$ the number of the scalar components of the vector $Y$, and $E^{k, \Xi}$ the $k_{\Xi}$-dimensional Euclideal space. If in the total probability formula

$$
f(y)=\int_{E^{k \Xi}} f(y / \xi) f(\xi) d \xi
$$

the conditional probability density function $f(y / \xi)$ is represented in the form

$$
f(y / \xi)=\frac{1}{\sqrt{(2 \pi)^{k Y}\left|d_{Y}\right|}} \exp \left\{-\frac{1}{2} \sum_{i=1}^{m} \sum_{j=1}^{m} \xi_{i}^{T} S_{i, j} \xi_{j}+\sum_{i=1}^{m} V_{i}^{T} \xi_{i}-\frac{1}{2} W\right\}
$$

where $S_{i, j}$ are the blocks of a block symmetric positive definite matrix $S=\left(S_{i, j}\right), i, j=\overline{1, m}, V_{i}^{T}$ are the blocks of a block matrix $V^{T}=\left(V_{i}^{T}\right), i=\overline{1, m}, W$ is a scalar, and the probability density function $f(\xi)$ is represented in the form

$$
\begin{gathered}
f(\xi)=\frac{1}{\sqrt{(2 \pi)^{k \Xi}\left|d_{\Xi}\right|}} \exp \left(-\frac{1}{2} \sum_{i=1}^{m} \sum_{j=1}^{m}\left(\xi_{i}-v_{\Xi, i}\right)^{T} d_{\Xi}^{i, j}\left(\xi_{j}-v_{\Xi, j}\right)\right)= \\
=\frac{1}{\sqrt{(2 \pi)^{k \Xi}\left|d_{\Xi}\right|}} \exp \left\{-\frac{1}{2} \sum_{i=1}^{m} \sum_{i=1}^{m} \xi_{i}^{T} d_{\Xi}^{i, j} \xi_{j}+\sum_{i=1}^{m} \sum_{j=1}^{m} v_{\Xi, i}^{T} d_{\Xi}^{i, j} \xi_{j}-\sum_{i=1}^{m} \sum_{j=1}^{m} \frac{1}{2} v_{\Xi, i}^{T} d \Xi v_{\Xi, j}^{i, j}\right\},
\end{gathered}
$$

where $d_{\Xi}^{i, j}$ are the blocks of a block symmetric positive definite matrix $d_{\Xi}^{-1}=\left(d_{\Xi}^{i, j}\right), i, j=\overline{1, m}, v v_{\Xi, i}^{T}$ are the blocks of a block matrix $v_{\Xi}^{T}=\left(v_{\Xi}^{T}, i\right), i=\overline{1, m}$, then integral (20) (the total probability formula) is defined by the following expression

$$
f(y)=\int_{E^{k \Xi}} f(y / \xi) f(\xi) d \xi=\frac{1}{\sqrt{(2 \pi)^{k Y}\left|d_{\Xi}\left\|d_{Y}\right\| A\right|}} \exp \left(\frac{1}{2} B^{T} A^{-1} B-\frac{1}{2} C\right)=
$$




$$
=\frac{1}{\sqrt{(2 \pi)^{k_{Y}}\left|d_{\Xi}\left\|d_{Y}\right\| A\right|}} \exp \left(\frac{1}{2} \sum_{i=1}^{m} \sum_{j=1}^{m} B_{i}^{T} A^{i, j} B_{j}-\frac{1}{2} C\right),
$$

where

$$
\begin{gathered}
A=\left(A_{i, j}\right)=\left(d_{\Xi}^{i, j}+S_{i, j}\right)=d \Xi{ }^{-1}+S, \quad i, j=\overline{1, m}, \\
B=\left(B_{i}\right)=\left(\sum_{j=1}^{m} d_{\Xi}^{i, j} v_{\Xi, j}+V_{i}\right)=d \Xi v_{\Xi}+V, \quad i=\overline{1, m}, \\
C=\sum_{i=1}^{m} \sum_{j=1}^{m} v_{\Xi, i}^{T} d_{\Xi}^{i, j} v_{\Xi, j}+W .
\end{gathered}
$$

P r o o f. Multiplying (21) by (22), we get the following expression of the integrated function in (20):

$$
f(y / \xi) f(\xi)=\frac{1}{\sqrt{(2 \pi)^{k Y+k \Xi}\left|d_{Y} \| d_{\Xi}\right|}} \exp \left\{-\frac{1}{2} \sum_{i=1}^{m} \sum_{j=1}^{m} \xi_{i}^{T} A_{i, j} \xi_{j}+\sum_{i=1}^{m} B_{i} \xi_{i}-\frac{1}{2} C\right\},
$$

where $A, B, C$ are determined by formulae (24), (25), (26). In order to integrate the received function we can use formula (19) and write the equality

$$
f(y)=\int_{E^{k \Xi}} f(y / \xi) f(\xi) d \xi=\frac{\sqrt{(2 \pi)^{k \Xi}\left|A^{-1}\right|}}{\sqrt{(2 \pi)^{k Y+k \Xi}\left|d_{Y} \| d_{\Xi}\right|}} \exp \left(\sum_{i=1}^{m} \frac{1}{2} B^{T} A^{-1} B-\frac{1}{2} C\right) .
$$

The proof is completed.

The functions $f(y / \xi)$ and $f(\xi)$ in total probability formula (20) are usually given not in the form of expressions (21), (22), but in the natural for the Gaussian distribution form. In this case, theorem 2 takes the form of theorem 3 , more convenient for the practical utilization.

The or e m 3. Let $\Xi^{T}=\left(\Xi_{1}^{T}, \Xi_{2}^{T}, \ldots, \Xi_{m}^{T}\right)$ be a random vector composed of vectors $\Xi_{1}^{T}, \Xi_{2}^{T}, \ldots, \Xi_{m}^{T}$, $k_{\Xi, i}$ the number of the scalar components of the vector $\Xi_{i}, i=\overline{1, m}, f(\xi)$ the probability density function of the vector $\Xi, k_{\Xi}=k_{\Xi, 1}+k_{\Xi, 2}+\ldots+k_{\Xi, m}$ the number of the scalar components of the vector $\Xi, f(y / \xi)$ the conditional probability density function of the random vector $Y, k_{Y}$ the number of the scalar components of the vector $Y$, and $E^{k \Xi}$ the $k_{\Xi^{-}}$dimensional Euclideal space. If in the total probability formula

$$
f(y)=\int_{E^{k \Xi}} f(y / \xi) f(\xi) d \xi
$$

the conditional probability density function $f(y / \xi)$ has the following form

$$
\begin{aligned}
& f(y / \xi)=\frac{1}{\sqrt{(2 \pi)^{k_{Y}}\left|d_{Y}\right|}} \exp \left(-\frac{1}{2}(y-h \xi)^{T} d_{Y}^{-1}(y-h \xi)\right)= \\
& =\frac{1}{\sqrt{(2 \pi)^{k_{Y}}\left|d_{Y}\right|}} \exp \left(-\frac{1}{2}\left(y-\sum_{i=1}^{m} h_{i} \xi_{i}\right)^{T} d_{Y}^{-1}\left(y-\sum_{i=1}^{m} h_{i} \xi_{i}\right)\right),
\end{aligned}
$$

where $h=\left(h_{1}, h_{2}, \ldots, h_{m}\right)$ is a matrix composed of $\left(k_{Y} \times k_{\Xi, j}\right)$-matrices (blocks) $h_{i}, i=\overline{1, m}$, and the probability density function $f(\xi)$ has the following form

$$
\begin{gathered}
f(\xi)=\frac{1}{\sqrt{(2 \pi)^{k \Xi}\left|d_{\Xi}\right|}} \exp \left(-\frac{1}{2}\left(\xi-v_{\Xi}\right)^{T} d \Xi\left(\xi-v_{\Xi}\right)\right)= \\
=\frac{1}{\sqrt{(2 \pi)^{k \Xi}\left|d_{\Xi}\right|}} \exp \left(-\frac{1}{2} \sum_{i=1}^{m} \sum_{j=1}^{m}\left(\xi_{i}-v_{\Xi, i}\right)^{T} d_{\Xi}^{i, j}\left(\xi_{j}-v_{\Xi, j}\right)\right),
\end{gathered}
$$


where $v_{\Xi, i}$ are blocks of the matrix $v_{\Xi}=\left(v_{\Xi, i}\right)$, corresponding to the blocks of the random vector $\Xi_{i}$ $i=\overline{1, m}, d_{\Xi}^{i, j}$ are the blocks of the symmetric positive definite matrix $d_{\Xi}^{-1}=\left(d_{\Xi}^{i, j}\right), i, j=\overline{1, m}$, then integral (20) (the total probability formula) is defined by the following expression:

$$
\begin{gathered}
f(y)=\int_{E^{k \Xi}} f(y / \xi) f(\xi) d \xi=\frac{1}{\sqrt{(2 \pi)^{k Y}\left|D_{Y}\right|}} \exp \left(-\frac{1}{2}\left(y-h v_{\Xi}\right)^{T} D_{Y}^{-1}\left(y-h v_{\Xi}\right)\right)= \\
=\frac{1}{\sqrt{(2 \pi)^{k Y}\left|D_{Y}\right|}} \exp \left(-\frac{1}{2}\left(y-\sum_{i=1}^{m} h_{i} v_{\Xi, i}\right)^{T} D_{Y}^{-1}\left(y-\sum_{i=1}^{m} h_{i} v_{\Xi, i}\right)\right),
\end{gathered}
$$

where

$$
D_{Y}=d_{Y}+h d_{\Xi} h^{T}=d_{Y}+\sum_{i=1}^{m} \sum_{j=1}^{m} h_{i} d_{\Xi, i, j} h_{j}^{T}
$$

This theorem is the generalization of the theorem proved in [7].

\section{The Bayes formula for the joint vector Gaussian distribution.}

Th e o r e $\mathrm{m} 4$ (the Bayes formula for the joint vector Gaussian distribution). Let $\Xi^{T}=\left(\Xi_{1}^{T}, \Xi_{2}^{T}, \ldots, \Xi_{m}^{T}\right)$ be a random vector composed of vectors $\Xi_{1}^{T}, \Xi_{2}^{T}, \ldots, \Xi_{m}^{T}, k_{\Xi, i}$ the number of the scalar components of the vector $\Xi_{i}, f(\xi)$ the probability density function of the vector $\Xi, k_{\Xi}=k_{\Xi, 1}+k_{\Xi, 2}+\ldots+k_{\Xi, m}$ the number of the scalar components of the vector $\Xi, f(y / \xi)$ the conditional probability density function of the random vector $Y$, and $k_{Y}$ the number of the scalar components of the vector $Y$. If in the Bayes formula

$$
f(\xi / y)=\frac{f(\xi) f(y / \xi)}{\int_{E^{k \Xi}} f(\xi) f(y / \xi) d \xi}
$$

the conditional probability density function $f(y / \xi)$ is represented in the form

$$
f(y / \xi)=\frac{1}{\sqrt{(2 \pi)^{k y}\left|d_{Y}\right|}} \exp \left\{-\frac{1}{2} \sum_{i=1}^{m} \sum_{j=1}^{m} \xi_{i}^{T} S_{i, j} \xi_{j}+\sum_{i=1}^{m} V_{i}^{T} \xi_{i}-\frac{1}{2} W\right\},
$$

where $S_{i, j}$ are the blocks of a block symmetric positive definite matrix $S=\left(S_{i, j}\right), i, j=\overline{1, m}, V_{i}^{T}$ is the blocks of a block matrix $V^{T}=\left(V_{i}^{T}\right), i=\overline{1, m}, W$ is a scalar, and the probability density function $f(\xi)$ is represented in the form

$$
\begin{gathered}
f(\xi)=\frac{1}{\sqrt{(2 \pi)^{k \Xi}\left|d_{\Xi}\right|}} \exp \left(-\frac{1}{2} \sum_{i=1}^{m} \sum_{j=1}^{m}\left(\xi_{i}-v_{\Xi, i}\right)^{T} d_{\Xi}^{i, j}\left(\xi_{j}-v_{\Xi, j}\right)\right)= \\
=\frac{1}{\sqrt{(2 \pi)^{k \Xi}\left|d_{\Xi}\right|}} \exp \left\{-\frac{1}{2} \sum_{i=1}^{m} \sum_{i=1}^{m} \xi_{i}^{T} d_{\Xi}^{i, j} \xi_{j}+\sum_{i=1}^{m} \sum_{j=1}^{m} v_{\Xi, i}^{T} d_{\Xi}^{i, j} \xi_{j}-\sum_{i=1}^{m} \sum_{j=1}^{m} \frac{1}{2} v_{\Xi, i}^{T} d_{\Xi}^{i, j} v_{\Xi, j}\right\},
\end{gathered}
$$

where $d_{\Xi}^{i, j}$ are the blocks of a block symmetric positive definite matrix $d_{\Xi}^{-1}=\left(d_{\Xi}^{i, j}\right), i, j=\overline{1, m}, v \stackrel{T}{\Xi, i}$ are the blocks of a block matrix $v_{\Xi}^{T}=\left(v_{\Xi}^{T}, i\right), i=\overline{1, m}$, then the posterior probability density function $f(\xi / y)$ of the random vector $\Xi$ defined by the Bayes formula (28), has the following form:

$$
\begin{gathered}
f(\xi / y)=\frac{1}{\sqrt{(2 \pi)^{k \Xi}\left|D_{\Xi}\right|}} \exp \left(-\frac{1}{2}\left(\xi-\mathrm{N}_{\Xi}\right)^{T} D_{\Xi}^{-1}\left(\xi-\mathrm{N}_{\Xi}\right)\right)= \\
=\frac{1}{\sqrt{(2 \pi)^{k \Xi}\left|D_{\Xi}\right|}} \exp \left(-\frac{1}{2} \sum_{i=1}^{m} \sum_{j=1}^{m}\left(\xi_{i}-\sum_{k=1}^{m} D_{\Xi, i, k} B_{k}\right)^{T} D_{\Xi}^{i, j}\left(\xi_{j}-\sum_{l=1}^{m} D_{\Xi, i, l} B_{l}\right)\right),
\end{gathered}
$$

where 


$$
\begin{gathered}
D_{\Xi}^{-1}=\left(D_{\Xi}^{i, j}\right)=\left(d_{\Xi}^{i, j}+S_{i, j}\right)=d_{\Xi}^{-1}+S, \quad i, j=\overline{1, m}, \\
B=\left(B_{i}\right)=\left(\sum_{j=1}^{m} d_{\Xi}^{i, j} v_{\Xi, j}+V_{i}\right)=d_{\Xi}^{-1} v_{\Xi}+V, \\
\mathrm{~N}_{\Xi}=\left(\mathrm{N}_{\Xi, i}\right)=D_{\Xi} B=\sum_{k=1}^{m} D_{\Xi, i, k} B_{k} .
\end{gathered}
$$

Proof. Let us note that the given theorem is formulated in the same conditions and notations as theorem 2. In this case the numerator of the Bayes formula (28) is defined by expression (27) and the denominator by the total probability formula (23). Dividing (27) by (23), we get the formula

$$
\begin{gathered}
f(\xi / y)=\frac{1}{\sqrt{(2 \pi)^{k \Xi}\left|\left(A_{1,1}^{(0)}\right)^{-1}\right| \cdots\left|\left(A_{m, m}^{(m-1)}\right)^{-1}\right|}} \times \\
\times \exp \left(-\frac{1}{2} \sum_{i=1}^{m} \sum_{j=1}^{m} \xi_{i}^{T} A_{i, j} \xi_{j}+\sum_{i=1}^{m} B_{i}^{T} \xi_{i}-\frac{1}{2} \sum_{i=1}^{m} \sum_{j=1}^{m} B_{i}^{T} A^{i, j} B_{j}\right),
\end{gathered}
$$

where $A$ and $B$ are defined by formulae (24), (25). Formula (31) can be written in the following form

$$
f(\xi / y)=\frac{1}{\sqrt{(2 \pi)^{k \Xi}\left|A^{-1}\right|}} \exp \left(-\frac{1}{2} \sum_{i=1}^{m} \sum_{j=1}^{m}\left(\xi_{i}-\mathrm{N}_{\Xi, i}\right)^{T} A_{i, j}\left(\xi_{j}-\mathrm{N}_{\Xi, j}\right)\right),
$$

where

$$
\mathrm{N}_{\Xi, i}=\sum_{j=1}^{m} A^{i, j} B_{j}
$$

We will be convinced in the equality between (31) and (32) by multiplication in (32) given expression (33).

The matrix $d_{\Xi}=\left(d_{\Xi, i, j}\right), \quad i, j=\overline{1, m}$, in formula (29) is the block priory variance-covariance matrix of the random vector $\Xi^{T}=\left(\Xi_{1}^{T}, \Xi_{2}^{T}, \ldots, \Xi_{m}^{T}\right)$, the matrices $d \Xi_{\Xi}^{i, j}$ are the elements (blocks) of the block inverse matrix $d_{\Xi}^{-1}=\left(d_{\Xi}^{i, j}\right), i, j=\overline{1, m}$, and the vector $v_{\Xi}^{T}=\left(v_{\Xi, 1}^{T}, v_{\Xi, 2}^{T}, \ldots, v_{\Xi, m}^{T}\right)$ is the block priory mathematical expectation of the random vector $\Xi^{T}=\left(\Xi_{1}^{T}, \Xi_{2}^{T}, \ldots, \Xi_{m}^{T}\right)$. The matrix $A=\left(A^{i, j}\right)$ in the Bayes formula (32) is the posterior variance-covariance matrix of the random vectors $\Xi\left(A^{i, j}=\operatorname{cov}\left(\Xi_{i}, \Xi_{j} / y\right)\right.$ that we denote now $D_{\Xi}=\left(D_{\Xi, i, j}\right)=A^{-1}=\left(A^{i, j}\right)$. The vector $\mathrm{N}_{\Xi}=\left(\mathrm{N}_{\Xi, i}\right)=\left(\sum_{j=1}^{m} A^{i, j} B_{j}\right)=\left(\sum_{j=1}^{m} D_{\Xi, i, j} B_{j}\right), i=\overline{1, m}$, in the Bayes formula (32) is the posterior mathematical expectation of the random vector $\Xi^{T}=\left(\Xi_{1}^{T}, \Xi_{2}^{T}, \ldots, \Xi_{m}^{T}\right) \quad\left(\mathrm{N}_{\Xi, i}=E\left(\Xi_{i} / y\right)\right)$.

This completes the proof of theorem 4.

4. Example. Multiple Bayesian regression. We consider an example of obtaining the Bayes estimations of the coefficients of the multiple regression function. Let $x^{T}=\left(x_{1}, x_{1}, \ldots, x_{n_{X}}\right)$ be the vector input variable of some regression object and the scalar output variable $Y$ of this object have the Gaussian probability density function

$$
f(y / x, \Xi)=\frac{1}{\sqrt{2 \pi d_{Y}}} \exp \left(-\frac{1}{2} d_{Y}^{-1}\left(y-\sum_{k=1}^{m} h_{k}^{T} \Xi_{k}\right)^{2}\right),
$$

where $\sum_{k=1}^{m} h_{k}^{T} \Xi_{k}$ is the regression function of $Y$ on $x$. In contrast to the classical case, we consider the case when the vector of the basis functions $h^{T}=\left(h_{1}^{T}, h_{2}^{T}, \ldots, h_{m}^{T}\right)$ contains not the scalar components, but is 
represented as a set of the vector components $h_{1}^{T}, h_{2}^{T}, \ldots, h_{m}^{T}, h_{i}^{T}=h_{i}^{T}(x), i=1,2, \ldots, m$. The vector $\Xi^{T}=\left(\Xi_{1}^{T}, \Xi_{2}^{T}, \ldots, \Xi_{m}^{T}\right)$ is the vector of the coefficients of the regression function which is represented, like the vector $h$, by a set of its vector components $\Xi_{1}^{T}, \Xi_{2}^{T}, \ldots, \Xi_{m}^{T}$. Each vector $\Xi_{i}$ has the $k_{\Xi_{i}}$ scalar components. Let the vector coefficients $\Xi_{1}, \Xi_{1}, \ldots, \Xi_{m}$ have the mean values $v_{\Xi_{1}}, v_{\Xi_{2}}, \ldots, v_{\Xi_{m}}$ and the covariance matrices $d_{\Xi, i, j}, i, j=1,2, \ldots, m$, so that $d_{\Xi}=\left(d_{\Xi, i, j}\right)$ is the block variance-covariance matrix of the vector $\Xi$. Let the vector $\Xi$ have the Gaussian probability density function:

$$
f(\xi)=\frac{1}{\sqrt{(2 \pi)^{k \Xi}\left|d_{\Xi}\right|}} \exp \left(-\frac{1}{2} \sum_{i=1}^{m} \sum_{j=1}^{m}\left(\xi_{i}-v_{\Xi, i}\right)^{T} d_{\Xi}^{i, j}\left(\xi_{j}-v_{\Xi, j}\right)\right), \quad k_{\Xi}=k_{\Xi_{1}}+\ldots+k_{\Xi_{m}},
$$

where $d_{\Xi}^{i, j}$ are the blocks of the matrix $d_{\Xi}^{-1}=\left(d_{\Xi}^{i, j}\right)$ inverse to the matrix $d_{\Xi}=\left(d_{\Xi, i, j}\right)$.

Let us formulate the task of finding the Bayes estimations $\bar{\Xi}_{k}$ of the coefficients $\Xi_{k}$ of the regression function $\sum_{k=1}^{m} h_{k}^{T} \Xi_{k}$ on the basis of independent measurements $\left(x_{1}, y_{1}\right),\left(x_{2}, y_{2}\right), \ldots,\left(x_{n}, y_{n}\right)$.

Let the regression function be the function of two variables $x_{1}, x_{2}$ in the following form:

$$
\varphi=\alpha+\beta_{1} x_{1}+\beta_{2} x_{2}+\gamma_{1} x_{1}^{2}+\gamma_{2} x_{2}^{2}+\gamma_{3} x_{1} x_{2},
$$

where $\alpha, \beta_{1}, \beta_{2}, \gamma_{1}, \gamma_{2}, \gamma_{3}$ are the unknown coefficients, and we wish to divide these coefficients into 3 vectors representing the intercept, linear, and quadratic parts of the regression function. Then we have to choose the following vectors of the coefficients:

$$
\Xi_{1}=\alpha, \quad \Xi_{2}^{T}=\left(\beta_{1}, \beta_{2}\right), \quad \Xi_{3}^{T}=\left(\gamma_{1}, \gamma_{2}, \gamma_{3}\right),
$$

and the following vectors of the basis functions:

$$
h_{1}=1, \quad h_{2}^{T}=\left(x_{1}, x_{2}\right), \quad h_{3}^{T}=\left(x_{1}^{2}, x_{2}^{2}, x_{1} x_{2}\right) .
$$

We will use theorem 4 for solving the task. The set of the measurements $y=\left(y_{\mu}\right), \mu=\overline{1, n}$, is the vector with following probability density function:

$$
f(\bar{y} / \bar{x}, \Xi)=\prod_{\mu=1}^{n} f\left(y_{\mu} / x_{\mu}, \Xi\right)=\frac{1}{\sqrt{(2 \pi)^{n_{Y}}\left|d_{Y}\right|}} \exp \left(-\frac{1}{2} \sum_{\mu=1}^{n} d_{Y}^{-1}\left(y_{\mu}-\sum_{j=1}^{m} h_{\mu, j}^{T} \Xi_{j}\right)^{2}\right) .
$$

Let us transform function (35) to the form

$$
f(\bar{y} / \bar{x}, \Xi)=\frac{1}{\sqrt{(2 \pi)^{n Y}\left|d_{Y}\right|}} \exp \left\{-\frac{1}{2} \sum_{i=1}^{m} \sum_{j=1}^{m} \xi_{i}^{T} S_{i, j} \xi_{j}+\sum_{j=1}^{m} V_{j}^{T} \xi_{j}-\frac{1}{2} W\right\},
$$

which is supposed in theorem 4. Since we have in (35)

$$
u_{\mu}=\left(\sum_{j=1}^{m} h_{\mu, j}^{T} \Xi_{j}\right)^{2}=\left(\sum_{i=1}^{m} \boldsymbol{\Xi}_{i}^{T} h_{\mu, i}\right)\left(\sum_{j=1}^{m} h_{\mu, j}^{T} \Xi_{j}\right)=\sum_{i=1}^{m} \sum_{j=1}^{m} \boldsymbol{\Xi}_{i}^{T} h_{\mu, i} h_{\mu, j}^{T} \Xi_{j}
$$

then

$$
\sum_{\mu=1}^{n} d_{y}^{-1} u_{\mu}=d_{Y}^{-1} \sum_{\mu=1}^{n} u_{\mu}=\sum_{i=1}^{2} \sum_{j=1}^{2} \Xi_{i}^{T}\left(d_{Y}^{-1} \sum_{\mu=1}^{n} h_{\mu, i} h_{\mu, j}^{T}\right) \Xi_{j}=\sum_{i=1}^{m} \sum_{j=1}^{m} \Xi_{i}^{T}\left(d_{Y}^{-1} S_{h h, i, j}\right) \Xi_{j}
$$

where

$$
S_{h h, i, j}=\sum_{\mu=1}^{n} h_{\mu, i} h_{\mu, j}^{T}, \quad S_{h h}=\left(S_{h h, i, j}\right)=\left(\sum_{\mu=1}^{n} h_{\mu, i} h_{\mu, j}^{T}\right), \quad i, j=\overline{1, m}
$$


In such a case we obtain the following expression for $S_{i, j}$ in (36):

$$
S_{i, j}=d_{Y}^{-1} S_{h h, i, j}=d_{Y}^{-1} \sum_{\mu=1}^{n} h_{\mu, i} h_{\mu, j}^{T}, \quad S=\left(S_{i, j}\right)=d_{Y}^{-1} S_{h h}, \quad i, j=\overline{1, m}
$$

Further, since in (35)

$$
\sum_{\mu=1}^{n} d_{Y}^{-1} y_{\mu} \sum_{j=1}^{m} h_{\mu, j}^{T} \Xi_{j}=\sum_{j=1}^{m}\left(d_{Y}^{-1} \sum_{\mu=1}^{n} y_{\mu} h_{\mu, j}^{T}\right) \Xi_{j}=\sum_{j=1}^{m} d_{Y}^{-1} S_{y h, j}^{T} \Xi_{j}
$$

where

$$
S_{y h, j}^{T}=\sum_{\mu=1}^{n} y_{\mu} h_{\mu, j}^{T}, \quad S_{y h}^{T}=\left(S_{y h, j}^{T}\right)=\left(\sum_{\mu=1}^{n} y_{\mu} h_{\mu, j}^{T}\right), \quad i, j=\overline{1, m}
$$

then we obtain the following expression for $V_{j}^{T}$ in (36):

$$
V_{j}^{T}=d_{Y}^{-1} S_{y h, j}^{T}=d_{Y}^{-1} \sum_{\mu=1}^{n} y_{\mu} h_{\mu, j}^{T}, \quad V^{T}=\left(V_{j}^{T}\right)=\left(d_{Y}^{-1} S_{y h, j}^{T}\right)=d_{Y}^{-1} S_{y h}^{T}, \quad j=\overline{1, m} .
$$

The probability density function $f(\xi)(34)$ is presented easily in the following form:

$$
f(\xi)=\frac{1}{\sqrt{(2 \pi)^{k \Xi}\left|d_{\Xi}\right|}} \exp \left(-\frac{1}{2} \sum_{i=1}^{m} \sum_{j=1}^{m} \xi_{i}^{T} d_{\Xi}^{i, j} \xi_{j}+\sum_{j=1}^{m}\left(\sum_{i=1}^{m} v_{\Xi, i}^{T} d_{\Xi}^{i, j}\right) \xi_{j}-\frac{1}{2} \sum_{i=1}^{m} \sum_{j=1}^{m} v_{\Xi, i}^{T} d_{\Xi}^{i, j} v_{\Xi, j}\right),
$$

supposed in theorem 4.

Thus, on the basis of theorem 4 (formula (30)), the joint posterior probability density function of the coefficients $\Xi_{1}, \Xi_{1}, \ldots, \Xi_{m}$ has the following form:

$$
\begin{gathered}
f(\xi / \bar{y}, \bar{x})=\frac{1}{\sqrt{(2 \pi)^{k \Xi}\left|D_{\Xi}\right|}} \exp \left(-\frac{1}{2}\left(\bar{\xi}-\mathrm{N}_{\Xi}\right)^{T} D_{\Xi}^{-1}\left(\bar{\xi}-\mathrm{N}_{\Xi}\right)\right)= \\
=\frac{1}{\sqrt{(2 \pi)^{k \Xi}\left|D_{\Xi}\right|}} \exp \left(-\frac{1}{2} \sum_{i=1}^{m} \sum_{j=1}^{m}\left(\xi_{i}-\sum_{k=1}^{m} D_{\Xi, i, k} B_{k}\right)^{T} D_{\Xi}^{i, j}\left(\xi_{j}-\sum_{l=1}^{m} D_{\Xi, j, l} B_{l}\right)\right),
\end{gathered}
$$

where

$$
\begin{gathered}
D_{\Xi}^{-1}=\left(D_{\Xi}^{i, j}\right)=\left(d_{\Xi}^{i, j}+d_{Y}^{-1} S_{h h, i, j}\right)=d_{\Xi}^{-1}+d_{Y}^{-1} S_{h h}, \quad S_{h h}=\left(S_{h h, i, j}\right)=\left(\sum_{\mu=1}^{n} h_{\mu, i} h_{\mu, j}^{T}\right), \quad i, j=\overline{1, m}, \\
B=\left(B_{i}\right)=\left(\sum_{j=1}^{m} d_{\Xi}^{i, j} v_{\Xi, j}+d_{Y}^{-1} S_{y h, i}\right)=d_{\Xi}^{-1} v_{\Xi}+d_{Y}^{-1} S_{y h}, \quad S_{y h}=\left(S_{y h, i}\right)=\left(\sum_{\mu=1}^{n} y_{\mu} h_{\mu, i}\right), \quad \mathrm{N}_{\Xi}=D_{\Xi} B .
\end{gathered}
$$

The $\mathrm{N}_{\Xi}$ and the $D_{\Xi}$, are the block posterior mathematical expectation and the block posterior variance-covariance matrix of the random vector $\Xi$ respectively.

The calculations are performed in the following order. The parameters of the unknown block vector parameter $\Xi$ distribution are given: the block variance-covariance matrix $d_{\Xi}=\left(d_{\Xi, i, j}\right), i, j=\overline{1, m}$, and the block vector of mathematical expectation $v_{\Xi}^{T}=\left(v_{\Xi, i}^{T}\right), i=\overline{1, m}$. Then the block matrix $d_{\Xi}^{-1}=\left(d_{\Xi}^{i, j}\right)$ inverse to the matrix $d_{\Xi}$ is calculated. Further, the block matrices $S_{h h}=\left(S_{h h, i, j}\right), S_{y h}=\left(S_{y h, j}\right), B=\left(B_{j}\right)=\left(d_{\Xi}^{-1} v_{\Xi}\right)+d_{Y}^{-1} S_{y h}, i=\overline{1, m}$, are calculated. After that the block matrix $D_{\Xi}^{-1}=\left(D_{\Xi}^{i, j}\right)=\left(d_{\Xi}^{i, j}+d_{Y}^{-1} S_{h h, i, j}\right), i, j=\overline{1, m}$, is formed and the inverse to it block matrix $D_{\Xi}=\left(D_{\Xi, i, j}\right), i, j=\overline{1, m}$, is calculated. Finally, the block vector of the posterior mathematical expectation 
$\mathrm{N}_{\Xi}=D_{\Xi} B$ is defined. For performing these operations we need the programs of transposing, multiplying and inverting of the block matrices.

Conclusion. The results obtained in this paper are aimed at solving the dual control problems formulated in works $[9,10]$. The sequence of the control actions in the dual control of the multivariate stochastic objects is defined by the functional equations of the dynamic programming [9], which contain the integrals like the integrals calculated in this article. One of the practical examples is the task of the optimal allowance distribution as the task of the dual control considered in work [10].

\section{References}

1. Wakefield J. Bayesian and Frequentist Regression Methods. Springer, 2013. 709 p. https://doi.org/10.1007/978-1-44190925-1

2. DeGroot M. H. Optimal Statistical Decisions. McGraw-Hill Inc., 1970. 489 p.

3. Zellner A. An Introduction to Bayesian Inference in Econometrics. John Wiley and Sons, Inc., 1971. 448 p.

4. Berger J. O. Statistical Decision Theory and Bayesian Analysis. New York, Springer-Verlag, 1985. 618 p. https://doi. org/10.1007/978-1-4757-4286-2

5. Fel'dbaum A. A. Optimal Control Systems. New York, London, Academic Press, 1965. 452 p.

6. Mukha V. S., Kako N. F. Integrals and integral transformations connected with vector Gaussian distribution. Vestsi Natsyianal'nai akademii navuk Belarusi. Seryia fizika-matematychnykh navuk = Proceedings of the National Academy of Sciences of Belarus. Physics and Mathematics series, 2019, vol. 55, no. 4, pp. 457-466. https://doi.org/10.29235/1561-24302019-55-4-457-466

7. Mukha V. S., Kako N. F. Total probability formula for vector Gaussian distributions. Doklady BGUIR, 2021, vol. 19, no. 2, pp. 58-64. https://doi.org/10.35596/1729-7648-2021-19-2-58-64

8. Gantmacher F. R. The Theory of Matrices. Vol. 1. New York, Chelsea Publ. Company, 1959. 374 p. https://doi. org/10.1126/science.131.3408.1216-a

9. Mukha V. S., Kako N. F. Dual Control of Multidimensional-matrix Stochastic Objects. Informatsionnye tekhnologii i sistemy 2019 (ITS 2019): Materialy mezhdunarodnoi konferentsii, BGUIR, Minsk, 30 oktyabrya 2019 g. [Information Technologies and Systems 2019 (ITS 2019): Proceeding of the International Conference, BSUIR, Minsk, 30th October 2019]. Minsk, 2019, pp. 236-237 (in Russian).

10. Mukha V. S., Kako N. F. Flat Problem of Allowance Distribution as Dual Control Problem. Informatsionnye tekhnologii i sistemy 2020 (ITS 2020): Materialy mezhdunarodnoi konferentsii, BGUIR, Minsk, Belarus', 18 noyabrya 2020 g. [Information Technologies and Systems 2020 (ITS 2020): Proceeding of the International Conference, BSUIR, Minsk, Belarus, 18th November 2020]. Minsk, 2020, pp. 195-196 (in Russian).

\section{Information about the authors}

Vladimir S. Mukha - Dr. Sc. (Engineering), Professor, Professor of the Department of Information Technologies of Automated Systems, Belarusian State University of Informatics and Radioelectronics (6, P. Brovka Str., 220013, Minsk, Republic of Belarus). E-mail: mukha@bsuir.by

Nancy Forat Kako - Postgraduate Student, Belarusian State University of Informatics and Radioelectronics (6, P. Brovka Str., 220013, Minsk, Republic of Belarus). E-mail: kako.nancy@gmail.com

\section{Информация об авторах}

Муха Владимир Степанович - доктор технических наук, профессор, профессор кафедры информационных технологий автоматизированных систем, Белорусский государственный университет информатики и радиоэлектроники (ул. П. Бровки, 6, 220013, г. Минск, Республика Беларусь). Е-mail: mukha@bsuir.by

Како Нэнси Форат - аспирант, Белорусский государственный университет информатики и радиоэлектроники (ул. П. Бровки, 6, 220013, г. Минск, Республика Беларусь). E-mail: kako.nancy@gmail.com 